\title{
Campos de Sintonía Somática Durante el Desarrollo Infantil
}

\author{
PATRICIA CORDELLA M. ${ }^{1}$
}

1. Profesor auxiliar Escuela de Medicina. Psiquiatra, terapeuta familiar. Departamento de Psiquiatría Pontificia Universidad Católica de Chile.

\begin{abstract}
\section{Somatic Syntonic Fields During Child Development}

A model is presented that shows the developmental importance of emotional dyadic modulation in children. This phenomenon occurs when two bodies that share the same time/space generate adjustment procedures in their nervous system that participate in the development of a stress-vulnerable or stressresilient phenotype. Contact modalities are the basis of the social brain, and agreement/disagreement behaviors are called intersubject spaces. These spaces are basically a field of somatic syntony that organizes the adrenergic tone of each participant, their pattern of comfort or discomfort and a somatic predisposition to a healthy lifestyle or risk of disease. To talk about a syntonic field implies the belief that two organisms can regulate each other on contact, impact each other's body beyond the emotional aspects. A new model is provided that includes other issues, explains possible and necessary social exchange required for development and suggests a way to evaluate the status of the body based on a social exchange, as seen in a routine pediatric consultation.
\end{abstract}

(Key words: Child development, somatic syntonic fields).

Rev Chil Pediatr 2009; 80 (5): 407-419

\section{RESUMEN}

Se presenta un modelo que da cuenta de la importancia que tiene durante el desarrollo del niño la modulación emocional diádica. Este fenómeno que ocurre cuando dos cuerpos están compartiendo el mismo espacio-tiempo va generando en su recurrencia procedimientos de ajuste en el sistema nervioso extenso que participan en la construcción de un fenotipo vulnerable o resilente al stress. Los modos de encuentro son la base del cerebro social, y los actos de acuerdo/desacuerdo generados entre los cuerpos son los llamados espacios intersubjetivos. Este espacio es básicamente un campo de sintonía somática que organiza los tonos adrenérgicos de cada uno de los participantes, sus patrones de bienestar o malestar y la predisposición somática a una vida saludable o con riesgo de enfermar. Hablar de un campo sintónico supone creer en la posibilidad que dos organismos vivos se regulan mutuamente en sus encuentros y que esto compromete el cuerpo de ambos, más allá de lo emocional. El objetivo de este artículo es exponer un modelo inédito que aporte otros ángulos; explicar el intercambio social posible y necesario para el desarrollo, y entregar sugerencias para evaluar el estado del soma en la consulta pediátrica habitual en el espacio de intercambio social.

(Palabras clave: Desarrollo infantil, campo sintónico somático).

Rev Chil Pediatr 2009; 80 (5): 407-419

Trabajo recibido el 17 de junio de 2009, devuelto para corregir el 27 de agosto de 2009, segunda versión el 14 de septiembre de 2009, aprobado para publicación el 21 de septiembre de 2009.

Correspondencia a:

Dra. Patricia Cordella M.

E-mail:mpcordella@gmail.com 


\section{Introducción}

La modulación de las funciones corporales implica activación y silencio de diversas zonas del genoma y está íntimamente relacionada con la dinámica de la fisiología que los otros individuos cercanos ofrecen a esa conexión.

La modulación busca equilibrios que acerquen el organismo a funcionamientos saludables manteniéndolo en estados facilitadores del aprendizaje experiencial, de modo que puedan asentarse los procedimientos fisiológicos generales que garantizan una plataforma biológica conveniente a los sucesos adaptativos en los cuales se desplegará ese individuo. Esta modulación ha sido estudiada desde las perspectivas de la teoría del apego ${ }^{1}$, la intersubjetividad ${ }^{2}$ y la función reguladora relacional temprana ${ }^{3}$.

La modulación del organismo se produce primariamente entre los cuerpos. Es la expresión de la red extensa del sistema nervioso la que a través de la percepción de otro podrá variar el funcionamiento de diversos órganos. La fisiología de un individuo es modificada por la de otro individuo. La madre ejerce un rol primordial dado por la desigualdad de competencias para la superviviencia. Será el contacto mimético el que le dará al hijo la posibilidad de transformar informaciones perceptuales en entidades fuera del tiempo y del espacio real, es decir en abstracciones, e irá permitiendo formas modulantes. Al conjunto de estas funciones de carácter físiológico se le ha llamado el "self" el que corresponde al carácter automodulatorio o autoregulatorio y que corresponde a un aprendizaje derivado del anterior.

En este escrito presentamos un modelo que supone la participación de dos organismos como formadores de un campo de sintonía somática. La modalización (modo de hacer-el estar/ser) de la fisiología se produce a través de activar o desactivar procesos fisiológicos mutuamente. El mayor impacto lo tendrán los miembros con menor experiencia moduladora que se presentan a la interacción sin modos establemente adquiridos. Tras reiteradas conexiones modulantes (modelizantes), el cuerpo almacenaría en memorias de procedimiento ${ }^{5}$ estos verdaderos diálogos fisiológicos y los reeditarían automáticamente en presencia de otros sujetos. Este acoplamiento estructural ${ }^{6}$ que se da en al menos dos individuos, no necesariamente de la misma especie, ocurre desde el momento en que la organización molecular toma las dinámicas de la vida. La particular interacción madre hijo temprana es muy intensa para ambos. El niño necesita protección, alimento y modulación somática y la mujer-madre se ha transformado para ser eficiente con ese hijo en su tarea a tal punto que hace un cambio somático que condiciona incluso la secreción de hormonas (ocitocina, vasopresina y prolactina) convirtiendo el cuerpo en un productor de leche y abrigo dispuesto a producir conductas de filiación. En esta línea es que se ha desarrollado la teoría del apego $^{7}$, la que basada en la etología describe y mide y categoriza las conductas de apego en seguro e inseguro como marcadores de las cualidades de la relación entre seres humanos. Los apegos seguros logran que durante el periodo de desarrollo se asienten esquemas emocionales-cognitivos que le permiten al niño explorar sobre una base segura ${ }^{8}$ aún en ausencia de la madre. El niño aprendería durante los primeros años de vida a recrear los estados de inhibición de la alerta que habían sido primero activados por la presencia de la madre. También la teoría de la intersubjetividad ha aportado con la idea de la bidereccionalidad y las influencias recíprocas desplazando el concepto vincular a algo dinámico y cambiante según el contexto que esta misma relación construye ${ }^{9}$. Lo mismo ha ocurrido con la matriz relacional que Mitchell ${ }^{10}$ plantea como campo de interacciones dentro del cual surge el individuo y pugna por relacionarse y expresarse.

El objetivo de este artículo es proponer un nuevo modelo de campos de sintonía somática durante el desarrollo infantil, que incluya aspectos previos y que aporte una forma de pensar que haga sentido en la clínica pediátrica.

\section{Los Campos de Sintonía Somática}

Se plantea que la modulación de las funciones corporales ocurre en un campo de sintonía somática. Este campo corresponde a un espacio-tiempo compartido en el cual se capturan recogen y combinan las tendencias circulantes. 
Un campo de sintonía puede ser comprendido utilizando los modelos de campo electromagnético, el que según la teoría de Maxwell, sería aquel que se crea con la circulación de una corriente oscilante circulando por un conductor. El conductor produce esta corriente y la hace circular al mismo tiempo. Estas oscilaciones, son causa y efecto de la dinámica. En el caso de la relación madre-hijo cada uno de ellos sería el conductor del otro.

El campo sintónico es una constante homeostática ya que oscila entre el acoplamiento y el desacoplamiento en forma alternante. Podríamos pensar que es un sistema diádico en permanente error y corrección fisiológico que permite el aprendizaje del tono logrado a través del contacto. Esta conexión entre dos cuerpos disímiles (madre, hijo) se logra tanto por operaciones de homologación, es decir ambos sujetos se igualan en sus funciones o por encaje, es decir, ambos sujetos se reparten funciones complementarias, pero diferenciadas. Así por ejemplo, si el niño se angustia y la madre se angustia estarán ambos en un campo sintónico simétrico, a diferencia de un campo sintónico complementario donde si el niño se angustia la madre lo calma con tranquilidad.

Para la creación de un campo de sintonía somática se requiere al menos una diada. Al inicio se trata de un indefenso y su protector que es ya un sobreviviente al medio, es como su maestro de supervivencia. La diada madrehijo es por definición una diada complementaria, sin embargo, hay campos sintónicos que se crean para que el niño aprenda de la madre asuntos tan importantes como la: atención, motivación, negociación, filiación, que hará simetrizándose y homologándose al funcionamiento fisiológico de la madre. Con el desarrollo el niño aprenderá a estar en campos sintónicos más complejos como por ejemplo la triada madrepadre-hijo, así llamada complejo de Edipo, como en otros grupos humanos: sus pares, su equipo deportivo su grupo de trabajo etc.

Panksepp ${ }^{11}$ ha descrito el funcionamiento básico del organismo en forma de circuitos reguladores que posiciona en el cerebro, pero cuyas aferencias se encuentran por todo el cuerpo. Son los llamados circuitos emocionales primarios que se establecen tempranamente en el desarrollo posiblemente con una arquitectura genética de base, pero que se estabilizan dentro de los primeros contactos generadores de estos campos sintónicos. Este investigador los ha delimitado a través de funciones, moléculas participantes y zonas cerebrales que se activan. Los sistemas de hambre/saciedad; alarma y procreación no son considerados circuitos emocionales aunque requieren de estos para lograr funcionar. En cambio, sí lo son las emociones básicas como: placer, búsqueda, asco, rabia, miedo. También serían circuitos emocionales básicos: la reproducción (representada en el deseo como motivación dirigida a un objetivo), la filiación (como aquellos patrones de reunión y separación que indican pertenencia a una casta), la capacidad de regulación del conflicto social a través de la demarcación de fronteras territoriales como de poder, estableciendo en esta última las jerarquías y dando los roles de dominio y sumisión; y los modos de aprendizaje a través de la simulación, juegos de imitación y la competencia.

Los campos de sintonía somática ocurren como un fenómeno de la conciencia destinado a producir objetividad, es decir consenso de realidad. La convivencia requiere de varios acuerdos para hacer de la especie una masa de individuos coordinados que supere sus capacidades individuales y genere funciones colectivas que incrementan la supervivencia y la calidad de vida.

\section{Campos Sintónicos como Organizadores Fisiológicos}

\section{¿Qué importancia tiene exponer esto para la salud y el desarrollo del niño?}

Los campos de sintonía somática son potentes organizadores de la acción, la emoción y la cognición porque se encuentran a la base de los procesos moleculares que generan los flujos de acceso y egreso celular. Los procedimientos de excitación e inhibición del ADN son modulados, como sabemos por el ambiente, pero este ambiente no es un ente abstracto, se trata de campos oscilatorios disponibles creados por la presencia de los otros. Un individuo aislado pudiera con sus sensores percibir peligro, sin 
embargo, si se encuentra en comunidad es posible que la creación del campo sintónico somático compartido lo haga percibir protección y no active las alarmas de daño inminente. La madre, que representa esta comunidad, no es un individuo cerrado en sí mismo, sino, a su vez, es parte de otros campos oscilatorios dentro de los cuales los más importantes son el campo que co-crea con su pareja y con su propia madre ${ }^{12}$. Los campos oscilatorios no son asuntos que ocurran en un punto del tiempo sino que contienen todos los tiempos y espacios experienciales de la madre con sus significativos vivos o muertos. Al campo sintónico confluyen tanto los aspectos biológicos como los biográficos de la madre y del niño. Ambos llegan al encuentro con una historia. La del niño más breve, porque tiene meses de vida intrauterina, aunque no por eso menos importante ya que puede haber estado sometido a stress por razones metabólicas que le han organizado un eje HPA (hipotálamopituitaria-adrenal) tónico y presto a reaccionar ${ }^{13}$ y la madre que es un individuo con sus formas fisiológicas de estar-con a lo que llamamos muy simplemente personalidad, pero que corresponde a sistemas de creencias y organización perceptual automática, poderosas formas de excluir e incluir ciertas informaciones que perturban el equilibrio somático.

Los campos de sintonía somática serían el correlato físico-molecular del llamado espacio intersubjetivo ${ }^{14}$. Estos campos son oscilantes en su estar-con y oscilantes en las fronteras del yo-tu y solo logran estabilizarse mucho mas tarde en el desarrollo permitiendo al niño sostener definiciones acerca del funcionamiento de si mismo sin intromisiones biológicas de los significantes afectivos. Es decir el niño se constituirá en un sujeto independiente, fisiológicamente hablando, cuando logre sostener su coherencia aún en presencia de otro significativamente importante en campos oscilatorios conflictivos.

Un campo sintónico somático es un espacio-tiempo donde ocurren las oscilaciones diádicas homologas y por eso sintónicas a través de un acoplamiento estructural, es decir ambas fisiologías se ajustan para lograr el mismo estado de activación y alerta. Como se trata de una interacción, los movimientos de cada miembro de la diada participan en la creación de estos campos de modo que uno y otro pueden copiárselos y con esto entrar en sintonía. Por ejemplo, si la madre quiere que el niño coma ella misma abrirá la boca y hará los movimientos de rotación del alimento en la boca, buscando que el niño la copie. Este es un nivel simple de sintonía que ocurre desde el momento de nacer cuando el niño copia gestos de la cara de adultos que se le acercan a la cuna ${ }^{15}$.

La semiosis (producción de signos) también se activará desde el inicio dándole valor emocional a la experiencia y es este valor el que activará los procesos primarios emocionales que vienen dispuestos genéticamente para ser prendidos como circuitos establecidos haciendo que las zonas genéticas pertinentes activen la producción de las proteínas que participarán en la sintonía molecular, ver figura 1. Es por esto que en realidad se trata de dimensiones que están imbricadas unas con otras sin una sucesión ni temporal ni categorial formando una figura llamada cinta de Moebius donde una cosa es la otra (figura 2 y tabla 1).

\section{El Modelo Del Campo Sintónico}

Se trata de comprender que en la interacción madre-hijo se activa la totalidad del self. Esta totalidad ha sido estudiada de diferentes ciencias lo que ha producido la idea de separación

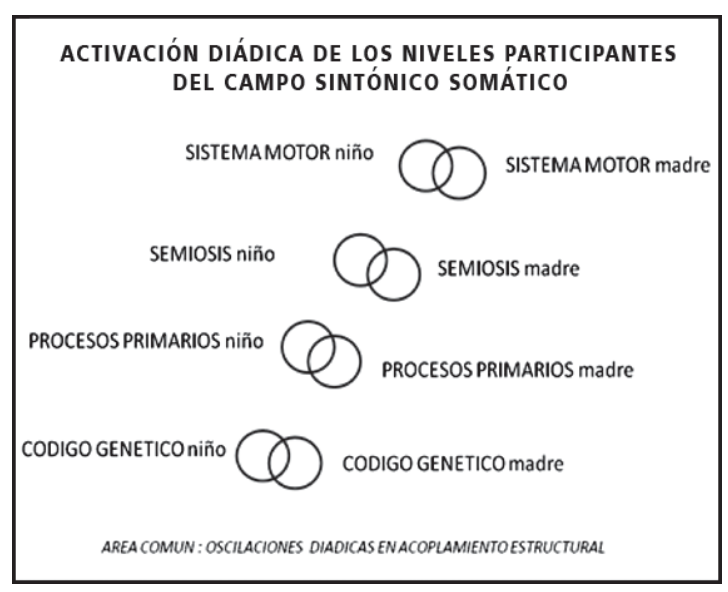

Figura 1. Campo sintónico: Activación mutua de niveles de procesamiento de la información. 
Tabla 1. Definiciones de las dimensiones participantes en el campo sintónico diádico

A. Código Genético: Información de la organización del ser vivo. Sólo cambia en millones a través de la evolución. Posiblemente por saltos evolutivos o adaptaciones al medio ambiente

B. Procesos Primarios: Se refiere a la activación de los circuitos primarios de Pankseep posiblemente de origen evolutivo que cumplen con procesos primarios de supervivencia

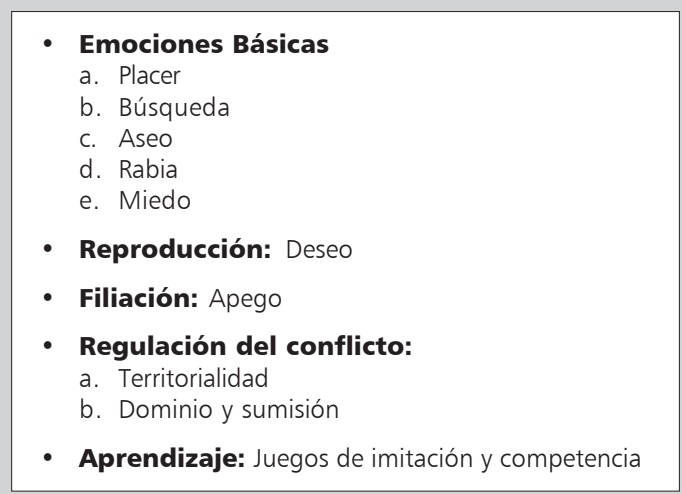

C. Semiosis: Proceso a través del cual se producen signos que permiten realizar las actividades cognitivas propias de la especie como seleccionar, conectar, transformar, geometrizar. Convencionalidad

D. Sistema Motor: Conectado tanto a sistema límbico como a corteza prefrontal que lo organiza, pasa a ser la vía del procesamiento perceptual. Figura 3. Las dimensiones activadas en el campo sintónico

de niveles. Sin embargo, son todos estos niveles que se encuentran activados en el campo se sintonía dispuesta a encontrarse o rechazarse produciendo conductas evidenciables a nivel motor que son las que podemos rescatar desde la observación clínica y más aún desde la

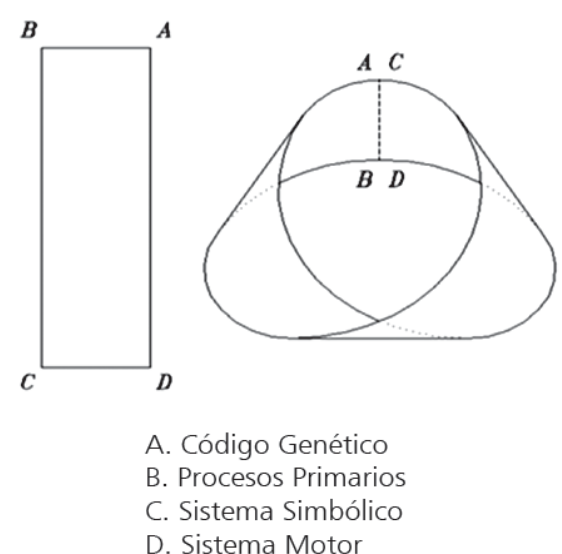

Figura 2. Modelo de campo sintónico: Los niveles activados: sistemas, códigos, procesos mantienen una relación tipo cinta de Moebius. microbservación videada. Los macroniveles activados son:

A. Código Genético: Corresponde a la actividad genésica. Aquella que genera información proteica y enlaza con esto la actividad de los otros niveles de análisis (nótese que se trata de niveles de análisis y no de funcionamiento).

B. Procesos Primarios: Corresponde a la activación de circuitos preestablecidos posiblemente antes de nacer y son la dotación acultural con la cual comenzamos a interactuar con el mundo y los otros. En el cuadro siguiente reorganizamos el modelo de Panksepp.

C. Sistema Simbólico: Corresponde a la construcción de significados que acortan el tiempo de reacción cuando se produce la emergencia de ciertos datos en el ambiente. El signo es el emergente intersubjetivo, es un acuerdo de significado. Este nivel implica reiteración de interacciones y se trata del proceso de culturalización.

D. Sistema Motor: Corresponde con el resultado visible de los procesos anteriores. Aquí hemos incluido tanto el gesto facial como el movimiento de desplazamiento. 
Evidencia de Campos Sintónicos:

El Uso del Microanálisis de Vídeos de Madre-hijo en Interacción

\section{¿Cómo podemos estudiar estos fenómenos de un modo que no sea invasivo y que esté más cerca de la clínica que de las matemáticas y la física?}

Para esto se ha creado una metodología de observación a la diada que propone videar las interacciones y registrar los eventos posibles de examinar ${ }^{16}$. Como estamos hablando de oscilaciones que ocurren en fracciones de segundos, al ojo humano se le escapan pormenores por lo cual junto con la posibilidad de registrar las imágenes y el sonido de la interacción existen software que hacen posible su estudio segmentado en millones de fotografías, tal como si se hiciera un scanner. El registro de la conducta motora evidente incluye especialmente la conducta motora de la cara. La gestualidad es un indicador emocional objetivo que da cuenta del estado límbico del sujeto ${ }^{17}$ y que produce evidentes cambios en el cerebro de quien percibe esta cara. Esto ha sido expuesto en múltiples investigaciones realizadas con imágenes funcionales del cerebro. También es importante en la conducta motora la danza que ocurre entre los cuerpos de los participantes. Danza que tiene ritmo (Lyon Ruth) y que puede ser gozosa o tensa, según el campo sintónico creado por ambos.

El sistema motor es una de las vías finales de la percepción y en términos de la comunicación pragmática podemos decir que un acto es el resultado de un proceso de elaboración hermenéutica tanto como una palabra. Es decir, cuando el niño decide mirar a la madre, inmediatamente antes de eso hubo cierta información que lo hizo interpretar el acto de la madre como abierto a recibir su mirada. El niño está siempre jugando en un proceso de interpretación de su madre. Lo que se ha llamado la teoría de la mente ya presente en primates ${ }^{18}$, es decir la capacidad de poder adelantarse a los actos del otro, al atribuirle pensamientos e intenciones. Siguiendo nuestro modelo diríamos que el niño es capaz de oscilar por momentos homologando la madre y desde allí desacoplarse para adelantarse a lo que probablemente ocurri- ría si él estuviera en esa oscilación. Esto parece complejo y efectivamente lo es, pero el niño desde que nace ya tiene estas capacidades. Por otra parte la madre también se encuentra permanentemente en estado de interpretación del niño ${ }^{19}$ es decir jugando con probabilidades, claro que la madre maneja información y experiencia que el niño no tiene incorporado. El niño así y todo es competente tanto en sintonizarse como en escapar de la madre, dos aspectos que aseguran su constitución como sujeto. Estar y no estar en contacto.

Los grupos que estudian lactantes han aportado con múltiples investigaciones durante más de treinta años logrando construir un corpus que podemos denominar Infant Research, esto ha sumado a la comprensión de los fenómenos relacionales en ese momento de la vida. La construcción de campos sintónicos pre-verbales suponen un cuerpo-en-acción como el lugar y procesamiento de la semiosis, es decir de la construcción de significados que permitan construir sentido.

Es entonces este cuerpo dispuesto a la conexión y la desconexión con otros que se va ajustando permanentemente para funcionar con sus cercanos, también llamados significativos porque operan como reguladores fisiológicos primarios y por lo tanto representan los más potentes moduladores de los campos sintónicos. Aunque se ha estudiado este ajuste temprano a través de la interacción videada de prácticas de crianza como comer, dormir o jugar nos parece evidente que alude a procesos moleculares conjuntos entre dos organismos que son los que crean estos campos oscilatorios y que modelan desde la percepción visual hasta los ritmos circadianos, limitando con esto el rango fisiológico de las vivencias y predisponiendo la fisiología a presentarse con cierta particular estabilidad. Es la oscilación dentro de ciertos rangos lo que permite hacer descripciones y anticiparse a las respuestas posibles y esta misma forma de respuesta biológica es lo que permite ciertas disfunciones y no otras. Por ejemplo, si en los sucesivos acoplamientos con la madre el niño aprende a inhibir sus aspectos pasivos y sobreactivarse como un modo de capturar la atención de una madre depresiva es posible que en el futuro le sea más difícil optar por la hipotonía, 
la lentitud, la hipotensión y el letargo necesarios, por ejemplo, para inducir el sueño o para estar con otro en estado receptivo.

Los campos oscilatorios son sistemas de dinámicas no lineales que vuelve a aparecer una y otra vez mostrando la misma trayectoria en el proceso de constituirse. Estos sistemas son estudiados por la matemática e intentan dar cuenta de estas organizaciones no lineales de la materia el tiempo y el espacio ${ }^{20}$, pero estas abstracciones se encuentran lejos del quehacer clínico.

Para nosotros, la observación de la interacción en micro-secuencias es ya un aspecto del estudio suficiente para ampliar la comprensión de cómo opera la lógica del encuentro entre estos dos seres vivos y de cómo ocurre la construcción de diversas desregulaciones que tomarán los nombres de las patologías médicas que conocemos.

Podemos decir entonces que el encuentro entre dos individuos es entendido, en esta nueva conceptualización, como la emergencia de un campo oscilatorio compartido. Procesos de negociación y acuerdo; colaboración y competencia; conexión y desconexión conforman desde los inicios el humus de la acción.

Los niños conectados con sus cercanos no pueden ser vistos ya como individuos, unidades cerradas que crecen siguiendo un programa determinado, como los primeros autores del desarrollo nos habían sugerido, aún nombrando contextos $^{21-23}$ sino como seres conectados que aprenden a modular y modelarse haciendo de estos procedimientos esquemas del saber estar-con (Stern).

Estos saber-hacer que son procedimientos de saber-estar (Stern) fijan rangos fisiológico que, evidenciados en acciones, nos informan de ajustes muy finos del acontecer molecular. Por ejemplo, un niño asustado debajo de las faldas de mamá, que no se atreve a mirar un tercero, nos indica altos índices de cortisol que están siendo compensados con la cercanía corporal materna porque no ha logrado desarrollar sistemas de autoregulación que le compensen la amenaza a la integridad que representa un desconocido. Si por ejemplo, este niño no es ayudado a autoregularse y esto se transforma en algo extendido en el tiempo, el niño podrá pre- sentar síntomas de esta desregulación que van tomando nombre de síndromes patológicos como son los trastornos de ansiedad de separación o más lejos, la depresión.

Esta nueva mirada sobre el desarrollo nos posiciona en un fenómeno complejo puesto que supone comprender lo que se ha llamado la enacción ${ }^{24}$ que es la interacción en tiempo presente de al menos dos seres vivos. No se trata de pensar para actuar sino de cómo la acción de un elemento de la diada va organizando en el momento mismo la acción del otro. En este sentido hablar de intersubjetividad nos desplaza a fenómenos más allá del apego.

\section{Apego, Intersubjetividad y Campos de Sintonía Oscilante}

La teoría del apego ha sido de utilidad para comprender como ocurre la regulación emocional en diadas tempranas, tanto humanas como animales. Nos ha ilustrado acerca de cómo se logra o como se pierde el bienestar somático con la presencia o ausencia de uno que representa protección, alimenta y mantiene el contacto necesario para la supervivencia afectiva.

Desde el apego conocemos la necesidad de ciertos marcadores de protección y contención como son: el tamaño del significante, sus olores, la temperatura y la textura. Recordemos la importancia del tacto en las primeras experiencias de Harlow $^{25}$ donde los monitos prefieren una madre de felpa por sobre una de alambre que les da leche. La cara del significante se reconoce como dato que organiza la teoría de la mente, es decir, reconocer en la cara del otro el estado afectivo daría información suficiente como para comprender sus procesos cognitivos. Sabemos que estos patrones de reunión y separación serán organizadores de futuras relaciones significativas y son posibles de medir a través del test de la situación extraña de Ainsworth en edad temprana ${ }^{26}$.

La teoría de la intersubjetividad ${ }^{27}$ intenta explicar cómo se organiza el sentido en y desde la superficie de los lactantes para alcanzar objetivos comunes, asunto de especial importancia en la coordinación de la especie. Cuando hablamos de intersubjetividad estamos en el 
mundo micro. Lo que ocurre en pequeños espacios/tiempos, mucho más cerca de la actividad eléctrica cerebral que de conductas evidentes. El apego corresponde en términos conceptuales a organizar el comportamiento en estructuras, que como tales, se repiten y modelan al ser humano queriendo dar cuenta de ciertos estados alterados de salud mental. Lyon Ruth $^{28}$, por ejemplo, ha estudiado el caso de los apegos ambivalentes que anteceden a conductas llamadas de borde (borderline personality) puesto que se trata de adolescentes que se encuentran frecuentemente al borde de la locura, al borde del peligro, al borde de la ley, al borde del peso sano, al borde de las normas sociales. Sin embargo, la idea de relacionar alteraciones del apego con precisas patologías mentales no resultó ser eficiente.

Por el contrario en la intersubjetividad se usa para pensar las relaciones en una lógica más cercana a la teoría de las probabilidades ya que no es posible no hacer hipótesis para comprender al otro y la hipótesis es sólo una posibilidad entre otras más que una inferencia. Poder adelantarse a lo que viene parece ser más bien una construcción de futuro que el futuro mismo. Es decir, es porque apuesto que sucederá tal cosa, hago los actos que están asociados a eso induciendo a que eso ocurra. Por ejemplo, si el niño cree que la madre lo mirará él se quedará quieto esperando esa mirada y aunque la madre estuviera distraída este pequeño estar de los ojos de su hijo en busca de los suyos podrá hacer que ella se sorprenda, le sonría y lo mire.

Los campos de sintonía oscilante encuentran su ritmo a través de organizar la percepción y la interpretación de la percepción de forma tal que le permita a los elementos de la diada hacer uso del repertorio de acciones que hacen posible sus acuerdos. Lo que oscila a simple vista es la conexión y desconexión de los cuerpos con el acuerdo y el desacuerdo de las partes. Si madre e hijo oscilan en su conexióndesconexión en forma paralela simétrica fluye una dinámica de acuerdo. Si por el contrario, uno de los miembros quiere cambiar de estado y busca conectarse y desconectarse y el otro se lo rechaza habrá un desacople del campo sintónico produciendo que el niño quede solo a cargo de reorganizar su campo perceptual, esto dependiendo del desarrollo logrado, puede producir mucha angustia o simplemente ocurrir. Si la madre o el hijo deciden variar el estado anterior y acuerdan hacerse complementarios entonces se entra en otro campo pero manteniendo la sintonía.

\section{Las Tareas Intersubjetivas del Campo Sintónico}

La tarea más importante de la intersubjetividad es crear objetividad, es decir, consenso entre dos sujetos. Este consenso de "que está pasando aquí-ahora", "que estamos haciendo y que queremos hacer" ocurre desde las primeras interacciones entre la madre y el hijo, las ya que necesitan acuerdos básicos como: cuanto rato dormir; comer; mojarse o que canciones, movimientos o ritmos son mejores para aquietarse.

La presencia de otro es un estímulo hermenéutico, es decir necesariamente el otro nos pone en función semiótica. Esto significa que el otro es siempre un estimulo a la interpretación y como tal a la construcción de signos. Puesto que el signo es algo que se pone en lugar de algo por algo ${ }^{29}$ es que podemos hacer las tareas de cognitivas de anticipar escenarios posibles y elegir el que nos acomode. Con un signo se puede pensar.

Un signo es una entidad dinámica tanto preconceptual como conceptual que tomando un elemento perceptual lo organizará dentro de una coherencia con sentido. Tanto el niño como la madre se encuentran compartiendo un campo de sintonía somática en la cual se producen oscilaciones de este quehacer somático. Entre las oscilaciones más básicas está el nivel de alerta dado por el nivel de alarma que se percibe en el medio ambiente incluido el otro. Este tono de alerta ambiental está dado por el eje HPA quien ha sido estimulado a su vez por el sistema límbico. Los signos más arcaicos por lo tanto son significantes de alarma que para un niño son en general índices del estado somático-emocional de la madre como el análisis de la musculatura de su cara. La madre operaría a través de estos y otros actos motores como 
modelo de la arquitectura cerebral especialmente del hemisferio derecho ${ }^{30}$ donde se asentarían los procesos más emocionales dejando para el izquierdo el quehacer lingüístico de desarrollo posterior.

Un signo requiere de un modelo de organización de la información y de enlaces de transformación. La madre traspasará modelos de interpretación y construcción de significado que el niño aprenderá a usar en la construcción de estos signos Este modelo se irá traspasando cotidianamente en las múltiples interacciones que sostendrá con su hijo/a. Reforzará, reprimirá, rechazará, ampliará, en fin, trasformará la experiencia del niño en coherencias sociales consensuadas dentro de la comunidad amplia a la que pertenece la madre.

Los signos le permitirán al niño realizar tareas cognitivas como hacer cadenas asociativas del tipo sujeto-predicado o causa-efecto haciendo con esto lo que llamamos la actividad simbólica y preparándolo para el lenguaje. Un símbolo es un signo elaborado, como decíamos, algo que está en vez de otra $\operatorname{cosa}^{31}$ y que no tiene con esa cosa ninguna relación de contigüidad o cualidad sino sólo de acuerdo cultural.

Un signo como la fiebre sería un índice de infección, si suponemos que la fiebre es desencadenada por trazas de microorganismos en el cerebro indicando que en esa percepción hay algo de aquello que la produce presente en el momento que el signo se hace evidente. No ocurre lo mismo con un cartel donde aparece una enfermera con el dedo índice sobre sus labios indicando silencio. Allí se trataría de un signo icono, puesto que la información que ese signo nos quiere entregar se parece en algo a la enfermera real. Más lejana es la relación que tiene la estrella impresa en la caja de los psicotrópicos controlados como el clonazepam donde sólo por consenso sabemos que eso significa receta retenida. Este es el caso del símbolo y sólo puede ser descifrado por un conocedor de los acuerdos de esa comunidad. El lenguaje se inscribe en este dominio, puesto que ciertos sonidos significan objetos, verbos (actos y sus tiempos) o cualidades y no existe relación más que el consenso entre ese sonido y lo que este indica.

Está claro que el niño en las primeras interacciones se maneja con signos cercanos a sus fuentes de origen. Son la cara de la madre, su olor, el tono de su voz las certezas que lo guían en la intersubjetividad. Es a través de estos índices que direcciona el próximo acto. La madre en cambio, suma a esto experiencias previas con otros hijos, relatos de sus amigas y familiares. Es decir el niño se enfrenta a la intersubjetividad con solo su intuición, es decir, su capacidad de decidir el sentido de la interacción dados ciertos signos primarios que le informan las intenciones de la madre.

La intersubjetividad es entonces un área común, un campo de interacciones dentro del cual surge el individuo se organiza e intenta tanto relacionarse (adaptarse) como expresarse (sostener su propia coherencia).

\section{Reguladores de la Interacción en el Campo Sintónico}

El gran regulador de la interacción es la empatía que se produce cuando un ser humano percibe e interpreta el cuerpo del otro, lo que comienza con la cara. Esta interpretación ocurre por un procedimiento de homologación donde el razonamientos es el siguiente: "ya que puedo copiar tu expresión facial, puedo sentir algo similar a lo que tú estás sintiendo y tener información de tu estado emocional, con esa información decido que hacer: a) Dejarla de lado: evitarla, rechazarla, negarla, ocultarla o prohibirla y con esto sostener transitoriamente el bienestar; b) Tomarla en cuenta: actuar para sostener o recuperar el bienestar".

La empatía es un fenómeno que va ocurriendo en interacción reciproca de modo que antes que se acabe un movimiento, el otro miembro de la diada ha completado el significado prediciendo hacia dónde va la intención de ese movimiento y está realizando otro. La interacción reciproca mantiene una homeostasis haciendo movimientos del tipo helicoidal que muestra la figura 3.

La empatía es la que permite se realice la coordinación, es decir, a través de usar sincronía, reciprocidad y pertinencia se lograrían realizar los ajustes, micro ajustes, siguiendo particulares ritmos que irían constituyendo patrones, 


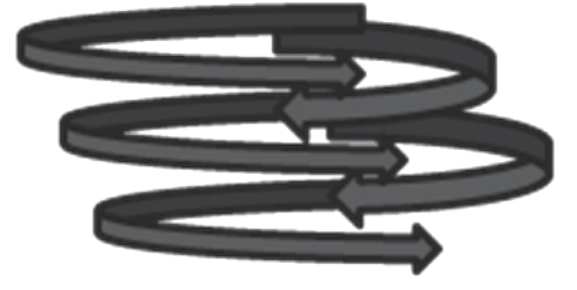

Figura 3. Campo sintónico: Forma en activa de interacción recíproca: la acción de uno estímula antes de terminar la del otro miembro de la diada.

que en el afán de aumentar la eficiencia se repetirían hasta que se produce un salto cualitativo y hay un cambio al que podemos llamar innovación. La innovación en los patrones interactivos está impulsada por las habilidades que va adquiriendo el niño en la maduración de sus sistemas somáticos y por la variabilidad ambiental.

La modulación ${ }^{32}$ transita a través de tonos adrenérgicos que activan, a su vez, patrones de reacción o solución, que son pautas muy tempranas y presentes en la etología. Dependiendo de cuáles son las señales que se ofrecen a la relación y de cómo son interpretadas por los miembros de la diada, se conforman patrones específicos con tonos adrenérgicos característicos. Se habla de tono adrenérgico pues éste representa el sistema de alarma y aunque no es el único que participaría en los campos sintónicos es el que se requiere modular o solucionar con urgencia pues puede representar riesgo vital.

Podemos ver en la figura 4 que hay dos polos uno caracterizado por el daño y otro por el amor. Desde cada uno de ellos podemos ver los grados de alerta adrenérgica. En la zona inferior de la curva que se presenta están los patrones interactivos de mejor funcionamiento diádico: el juego y la colaboración, pudiendo ambos producir innovación es decir hacer saltar el sistema hacia cambios, favoreciendo el desarrollo de la diada y especialmente del niño.

La regulación del campo somático sintónico ocurre tanto en el tiempo a través de los proce- sos de sincronía, como en el espacio a través de los movimientos y el perimetraje de espacios novedosos ${ }^{33}$. En el espacio/tiempo en cambio es donde ocurre la coordinación.

La sincronía aparece bajo las formas de ocurrencias simultáneas, patrones de interacción y secuencias preestablecidas de actos como si fueran rituales preconsabidos, una especie de baile co-creado por los miembros de la diada. Los ritmos en este baile están marcados por la conexión y desconexión de las partes. El acuerdo rítmico es un dialogo que ocurre sin palabras y que pacta un convenio.

La coordinación ocurre entre la propuesta y la respuesta de uno y otro miembro de la diada con la interpretación y el acuerdo correspondientes mediados por la capacidad de homologación motora o imitación a través de las llamadas neuronas espejo ${ }^{34}$ que serían un conjunto de zonas cerebrales asociadas a actos motores que se activan cuando vemos a otros realizar esos actos motores.

Cada uno de los miembros de la diada opera como regulador ${ }^{35}$ somático del otro a través de las acciones conjuntas y cada diada crea formas de intersubjetividad ${ }^{36}$ a través de procesos de encaje y enacción que sintonizan a los participantes.

La negociación es una actividad que se inicia desde los primeros encuentros y que requie-

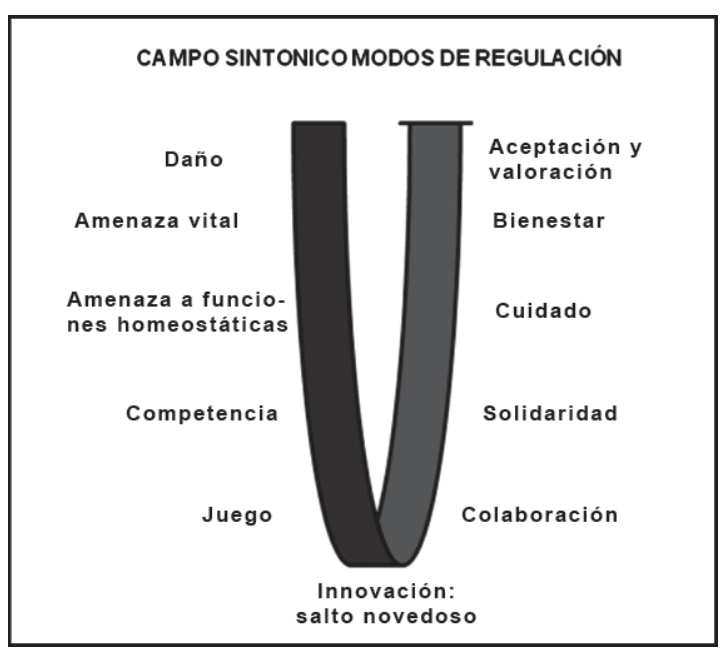

Figura 4. Activación de patrones interactivos y modulación del tono adrenérgico. 
re las capacidades de: atención, corte y secuencialidad, sensibilidad y semiosis como reguladores del campo sintónico.

La atención es la capacidad de sostener un foco para capturar la información necesaria para el acto que sigue. El corte y la secuencialidad son las capacidades de hacer giros de sentido que modifican el acto y la capacidad de enlazar un evento con otro produciendo la sensación de continuidad. La sensibilidad es la capacidad de empatía es decir cómo ponerse en el lugar fisiológico del otro para adelantarse al próximo acto de modo de sostener la propia intencionalidad. La semiosis es la capacidad de significar que cada parte utiliza para regular al otro hacia un objetivo deseado.

\section{Diagnóstico Clínico del Campo de Sintonía Somática}

Evaluar los campos sintónicos prevalentes nos da una idea de cómo está ocurriendo la modulación afectiva que la madre logra con su hijo. Es posible que en ciertos momentos la interacción sea competitiva y no por eso sea ése el campo prevalente. Será una oportunidad para preguntarle a la madre como ocurre habitualmente. Las interacciones cotidianas como comer, vestirse, bañarse, controlar esfínteres o más tarde hacer las tareas, organizar la mochila son los temas que organizan campos sintónicos recurrentes. gún:

El campo sintónico puede ser evaluado se-

1) Observación de la interacción que sostiene la madre con el niño mientras realiza tareas cotidianas como vestirlo, lavarlo, acomodarlo, hacerlo dormir, darle de comer, abrigarlo etc. Estas prácticas cotidianas con el hijo se pueden clasificar entre: del lado de la valoración o el daño. Por el primero las conductas serán de colaboración y la sensación mutua de bienestar. Por el otro, en cambio, habrá competencia y amenazas y la sensación estará más cerca de la rabia y el rechazo. Como una forma de simplificar haremos un cuadro comparativo entre estas dos formas de presentarse a la observación de la interacción diádica. El colaborativo es aquel donde el encuentro su-
Tabla 2.

\begin{tabular}{lll}
\hline $\begin{array}{l}\text { Campo } \\
\text { sintónico }\end{array}$ & Colaborativo & Competitivo \\
\hline Movimientos & Ritmo acoplado & Arritmias \\
Gestos faciales & Sonrisas abiertas & Muecas \\
Vocalizaciones & Suaves, gorgogeos & Duras, llanto, gritos \\
Palabras & Cariñosas & Cortantes \\
Atención & Flotante & Focalizante \\
Espacio & Variable & Tiende a fijarse \\
Inclusión de & Posible & Difícil \\
terceros & & \\
\hline
\end{tabular}

cede con tranquilidad, ritmos acoplados, vocalizaciones suaves o gozosas, la madre utiliza un lenguaje cariñoso (si es pequeño, el baby talk) donde se permite entrar y salir de la interacción sin producir quiebres cargados de angustia por parte de ninguno de los miembros. El espacio es variable según las necesidades que se estén dando el minuto y un tercero es incluido sin dificultad. En el campo sintónico competitivo hay afectos hostiles, los movimientos son arrítmicos, entrecortados, la gestualidad facial muestra muecas, es decir rigideces y las vocalizaciones incluyen protestas; las palabras son de tono agrio y la atención se sostiene sin posibilidad de salir del campo sintónico competitivo cuando éste ya ha pasado a las intensidades de amenaza o real daño en el cuerpo (tabla 2).

2) Descripción materna de su sentir la relación: Preguntas directas a la madre de cómo se siente en la relación con su hijo: cómoda/incómoda; tranquila/inquieta; angustiada o alegre. El campo sintónico prevalente entre ambos va dando una definición de la relación. La madre se siente competente si la relación es fluida e incompetente si no se logra fácilmente el acuerdo con el niño. Como el campo es construido por la sensación de ambos es posible que ambos puedan sentir similar. La madre da cuenta verbal de lo que ocurre en el campo sintónico y el niño da cuenta somática a través de la desregulación somática que va desde molestias vagas, incomodidad y puede llegar incluso a la enfermedad.

3) Historia somática del hijo: La desregulación emocional produce niveles de cortisol elevado $^{37,38}$ y con esto alteraciones en el SNA 
e incluso en el sistema inmune todo lo cual determina múltiples cuadros que por su intensidad y compromiso llegan a la consulta pediátrica.

\section{Conclusión}

Se presenta un modelo que permite re- pensar el niño como un sujeto emergente desde un campo sintónico co-creado con al menos otro sujeto con el cual deberá negociar las oscilaciones fisiológicas que los organizan y predisponen a estar operando de forma tal que se acerque más al polo de la salud o de la enfermedad .

El concepto de mente como el asiento de procesos cognitivos y afectivos apoyados en el tejido nervioso como sostén material, no es suficiente para comprender los fenómenos del desarrollo. Es todo el cuerpo el que participa y conforma la interacción. Con todo el cuerpo se conoce; con todo el cuerpo se comunica; con el todo el cuerpo se acepta o rechaza la propuesta del otro. Es con todo el cuerpo que se lee y escribe al otro en su futuro actuar. El cuerpo en su totalidad es el sostén del significado ${ }^{39}$, dejando al lenguaje un área más restringida y posterior en el desarrollo aunque ya modelizada por estos primeros procesos de significación.

Algunos estarán tentados a decir que justamente lo que se negocia en el espacio intersubjetivo es una mente común la llamada mente compartida, es decir, la coordinación fisiológica que se realiza través de "correspondencias" o matching de acciones del tipo: miradas que se sostienen; sonrisas mutuas, movimientos concomitantes acoplados o simétricos; ritmo; prosodia, proxemia y contacto corporal así como objetivos compartidos harán oscilar el cuerpo de una u otra forma, conjugando espacio y tiempo en un campo común. Es la fusión de ambas fisiologías en una sintonía común la que inaugura las estrategias comunitarias de supervivencia.

Como médicos podemos comprender estas operaciones y facilitar procesos más sanos a través de diagnósticos oportunos de oscilaciones aberrantes. Estos podrían ser realizados a través de la observación clínica o más objetivamente desde el uso de herramientas como el video y el micro análisis de las interacciones.
La interacción es lo explicito lo evidente, lo medible y objetivable de la vida fisiológica en el campo de oscilaciones de ambos participantes.

\section{Referencias}

1.- Bowlby J: Vínculos afectivos: formación, desarrollo y pérdida. Madrid, Ed. Morata, 1976.

2.- Stolorow R, Atwood G, Brandchaft B: The intersubjective perspective. New Jersey, Edit. Jason Aronson Inc. Northvale, 1994.

3.- Gross James J: Handbook of Emotion Regulation. New York-London, Guilford Press, 2007.

4.- Kohut H: La restauración del sí-mismo. México, Paidos, 1977.

5.- Lyon RK: El inconsciente bipersonal: el diálogo intersubjetivo, la representación relacional actuada y la emergencia de nuevas formas de organización relacional. Aperturas Psicoanalíticas, www.aperturas.org (4). 2000.

6.- Maturana H, Varela F: El árbol del conocimiento. Santiago de Chile, Ed. Universitaria, 1989.

7.- Bowlby J: Vínculos afectivos: formación, desarrollo y pérdida. Madrid, Ed. Morata, 1976.

8.- Mahaler M: El nacimiento del infante humano. B. Aires. Ed Marymar, 1975.

9.- Lyon R, Solomon J: Procedures for identifying infants as isorganized/disori-ented during the Ainsworth Strange Situation. In: Attachment in the Preschool Years: Theory, Research and Intervention, eds. M. Greenberg, D. Cicchetti, \& E. M. Cummings. 1990, Chicago, University of Chicago Press.

10.- Mitchell SA: Conceptos relacionales en psicoanálisis. Barcelona, Siglo veintiuno, 1993.

11.- Panksepp J: Toward a general psychobiological theory of emotions. Behavioral and Brain. Science 1982; 5: 407-68.

12.- Stern D: La constelación maternal. B. Aires, Ed. Paidos, 1997.

13.- Gunnar MR, Donzella B: Social regulation of the cortisol levels in early human development. Psychoneuroendocrinology 2002; 27: 1-2: 199-220.

14.- Trevarthen $C$ : First things ûrst: infants make good use of the sympathetic rhythm of imitation, without reason or language. Journal of Child Psychotherapy 2005; 31; 1: 91-113.

15.- Meltzoff AN, Moore MK: Explaining facial imitation: a theoretical model. Early Development and Parenting 1997; 6: 179-192. 
16.- Beebe B: Brief mother-infant treatment: psychoanalytically informed video. Feedback infant mental health journal 2003; 24 (1): 24-52.

17.- Ekman P: Social Psychophysiology: A Sourcebook, 1983, New York, J. T. Cacioppo \& R. E. Petty Eds. The Guilford Press.

18.- Tomasello M, Carpenter M, Liszkowski U: A New Look at Infant Pointing. Child Development 2007; 78; 3: 705-22.

19.- Fonagy P, Target M: Mentalization and the Changing Aims of Child Psychoanalysis. Psychoanalytic Dialogues 1998; 8: 87-114.

20.- Petitot J: Special issue: Neurogeometry and visual perception. J Physiol (París) 2003; 97: 93-7.

21.- Klein M: Psiconálisis del desarrollo temprano: contribuciones al psicoanálisis. Horme. Breviarios psicoanalíticos. B. Aires. Editorial Paidos, 1986.

22.- Erikson E: Infancia y sociedad. Buenos Aires HormePaidos, 1986.

23.- Piaget J: La formación del símbolo en el niño. México. Fondo de cultura económica, 1961.

24.- Varela F: El fenómeno de la vida. Santiago de Chile, Dolmen Ediciones, 2000.

25.- Harlow H: El amor en las crías de monos. En: Psicobiología Evolutiva. Selección del Scientific American. 1976 Barcelona Ed. Fontanella, 1959.

26.- Ainsworth MD, Blehar MC: Waters Everett Wall Sally Patterns of attachment A Psychological Study of the Strange Situation 1978, New Jersey Lawrence Erlbaum Associates, publishers hillsdale.

27.- Trevarthen $C$ : The foundations of intersubjectivity: Development of interpersonal and cooperative understanding in infants. In: The Social Foundations of Language and Thought, (1980), ed. D. Olson. New York. Norton.
28.- Lyon-Ruth K: The Interface Between Attachment and Intersubjectivity: Perspective from the Longitudinal Study of Disorganized Attachment. Psychoanalytic Inquiry: A Topical Journal for Mental Health Professionals 2006 ; 26, 4: 595-616.

29.- Peirce Ch: La lógica considerada como semiótica. Madrid, Edición de Sara Barrena Biblioteca Nueva Madrid, 2007.

30.- Schore A: Affect Dysregulation and Disorders of the Self. 2003a. New York: W. W. Norton.

31.- Eco U: La struttura assente. Milano, Bompiani. Tascabili, 1996.

32.- Fonagy P, Gergely G, Jurist E, Target M: Affect Regulation, Mentalization, and the Development of the Self. New York. Other Press, 2002.

33.- Koh H, Glaser E, Flandin G, Butterworth B, Maki A, Delpy $D F$, et al: Functional optical signal analysis: a software tool for near-infrared spectroscopy data processing incorporating statistical parametric mapping. J Biomedical Optics 2007; 12; 6: 064010-01-064010-13.

34.- Rizzolatti G, Craighero L: The mirror neuron system. Ann Rev Neurosi 2004; 27: 169-92.

35.- Tronick E: Dyadically expanded states of consciousness and the processof therapeutic change. Infant Mental Health Journal 1998; 19; 3: 290-9.

36.- Bebee B: Forms of Intersubjectivity in Infant Research and Adult Treatment. New York, Other Press, 2005.

37.- Pally $R$ : Emotional Processing: The Mind-Body Connection. International Journal of Psycho-Analysis 1998; 78: 349-62.

38.- Gunnar M, Quevedo K: The Neurobiology of Stress and Development. Annu Rev Psychol 2007; 58: 145-73.

39.- Violi P: Beyond the body: towards a full embodied semiosis. In R. Dirven, R.Frank eds., Body, Language and Mind, 2001, Mouton de Gruyter. Berlín. 